\title{
COMMENTARY/
}

\section{OVERAEAING THE ORACIES}

\section{by Bernard Dixon}

$F^{o}$ or those in need, one of the most deeply appreciated products of genetic tinkering must be recombinant human erythropoietin. Research over the past four years has shown that it corrects the anemia suffered by patients with end-stage kidney disease and thereby decreases the dangers of infection which are associated with blood transfusion and overload of iron. Earlier this year, major studies reported in the Journal of the American Medical Association (1990, 263:825) and the British Medical Journal $(1990,300: 573)$ also revealed that recombinant EPO greatly enhances the quality of life enjoyed by patients undergoing regular hemodialysis. It reduces fatigue, cornbats physical symptoms, and brings marked improvements in mood and exercise tolerance. A wide range of parameters, from sex life to sleep and eating behaviors, all show positive effects.

Given the profound physiological disturbances which are associated with dialysis, and the misery and depression that can afflict even the most stoic of patients who require weekly treatment on a kidney machine, these are weighty advances. Until now, hemodialysis has remained a therapy whose original technological wizardry has not been matched by advances in comfort and acceptance for the patient. Biotechnology has now provided the next real step forward.

It's strange, then, that erythropoietin was not cited at all in a major Delphi poll carried out just over a decade ago to forecast future research breakthroughs considered likely to flow from recombinant DNA research up to and beyond the year 2000. Organized by staff at CarnegieMellon University in Pittsburgh, PA, the crystal ball exercise harnessed the combined cerebration of 22 experts in medicine, agriculture, industry, basic research, government, and environmental policy. Reporting their findings in Futures $(1981,13: 1228)$, Shelby Stewman and his colleagues highlighted many potential advances in genetic manipulation, and cited as one of the most striking conclusions of their poll "the possible nearness, if not immediacy, with which many of these breakthroughs may occur."

Post-mortems on Delphi polls are surprisingly rare. Perhaps the participants in such projects, encouraged to think imaginatively and speculate boldly at the outset, are unwilling to review their prognostications in the sober light of later reality. Or maybe the fault lies with the companies and institutes that finance work of this sort. Do they simply lose interest in the accuracy of predictions for which they shell out substantial sums of hard-earned money? Or are they reluctant at a later date to risk the discovery that what they paid for was no more than a mixture of science fiction, naiveté, and statements of the obvious?

Whatever the explanation for past neglect, Delphi polls ought to be assessed for their insight and utility, and a decade is an appropriate interval after which to do so. The objective should not simply be to giggle over spectacular errors made by apparently well-informed forecasters. More important is the opportunity to consider improvements in the methodology of the Delphi technique, which seems not to have altered significantly since it was first devised during the late 1960s.

The Pittsburgh recombinant DNA study consisted of three rounds. The first was an open-ended trawl in which 22 experts identified future benefits in basic knowledge and in medicine, agriculture, and industry. Then the same panelists specified the years in which they believed there was a 50 percent- or 90 percent-probability of each breakthrough being realised. In the third round, participants were shown the distribution of these time estimates and allowed to revise their initial forecasts.

In general, the results stand up well to review, although, with hindsight, the wording of some topics appears to have been unhelpfully imprecise. The mean dates for $\mathbf{5 0}$ percent- and 90 percent-probability estimates in the case of screening for those genes responsible for birth defects were 1985 and 1990, respectively. The corresponding years for the development of bacteria for waste treatment and pollution control were 1984 and 1989 , and those for single cell protein were 1982 and 1987 . Nineteen ninety and 2000 emerged as the mean dates for new crops resistant to predators; dates for "understanding the immunological process" were 1984 and 1991.

Panelists also prophesied when six specific developments would reach the marketplace. Here the 50 percentand 90 precent-probability estimates were 2000 and 2045 for gene therapy, and 1985 and 1994 for effluent scavenging bacteria. The corresponding years for "petrochemical substitutes such as pesticides" were 1985 and 1995. Appropriately early dates (1983 and 1984) were determined for genetically engineered human insulin. Reflecting the consensus at the time, the most frequently mentioned agricultural advance was nitrogen fixation. The Delphi pollsters gave 1985 and 1995 as their 50 percent- and 90 percent-probability guestimates for the development of new nitrogen-fixing plants, and 1990 and 2000 as the years when they would become commercially available.

What Stewman and his collaborators did not do was to resolve all of the first- and second-round questions about possible breakthroughs into their component partsthose of technical feasibility and those of actuality in the real world. True, several panelists mentioned the NIH guidelines as constraints on research, and in six (out of 44) cases we can compare the dates forecast for particular innovations with those given for their commercial exploitation. But in a field so deeply enmeshed with regulatory, political, and social factors, it would have been highly instructive to ask the 22 experts to consider consciously and separately both the chances of developments being feasible on scientific grounds, and the likelihood of those innovations being fostered or hindered in the real world.

It's time, I think, for a thorough re-think concerning the rationale and protocols of the Delphi poll. 